\title{
Serum Fibroblast Growth Factor 21 Level Is Associated with Aortic Stiffness in Patients on Maintenance Hemodialysis
}

\author{
Liang-Te Chiu, ${ }^{1}$ Chi-Di Hung, ${ }^{2}$ Lin Lin, ${ }^{3}$ Yu-Li Lin $\mathbb{D}^{1,2}$ and Bang-Gee Hsu ${ }^{1,2}$ \\ ${ }^{1}$ Division of Nephrology, Hualien Tzu Chi Hospital, Buddhist Tzu Chi Medical Foundation, Hualien, Taiwan \\ ${ }^{2}$ School of Medicine, Tzu Chi University, Hualien, Taiwan \\ ${ }^{3}$ Division of Gastroenterology, Hualien Tzu Chi Hospital, Buddhist Tzu Chi Medical Foundation, Hualien, Taiwan \\ Correspondence should be addressed to Yu-Li Lin; nomo8931126@gmail.com and Bang-Gee Hsu; gee.lily@msa.hinet.net
}

Received 30 September 2021; Revised 2 January 2022; Accepted 17 January 2022; Published 11 February 2022

Academic Editor: Lanfranco D’Elia

Copyright (c) 2022 Liang-Te Chiu et al. This is an open access article distributed under the Creative Commons Attribution License, which permits unrestricted use, distribution, and reproduction in any medium, provided the original work is properly cited.

Background. Fibroblast growth factor 21 (FGF-21) is a hormone that regulates glucose and lipid metabolism. High serum FGF-21 levels are associated with carotid atherosclerosis and coronary artery disease. This cross-sectional study aimed to assess the relationship between serum FGF-21 levels and carotid-femoral pulse wave velocity (cfPWV) in patients on maintenance hemodialysis (HD). Methods. Blood samples and baseline characteristics were collected from 130 HD patients. Serum FGF-21 concentrations were measured with an enzyme-linked immunosorbent assay kit. Aortic stiffness was defined as a carotid-femoral pulse wave velocity (cfPWV) of more than $10 \mathrm{~m} / \mathrm{s}$. Results. Of the $130 \mathrm{HD}$ patients, aortic stiffness was diagnosed in 54 (41.5\%). Serum FGF-21 levels were significantly higher in those with aortic stiffness than those without $(P<0.001)$. The FGF-21 level was independently associated with aortic stiffness (odds ratio (OR): 1.008; 95\% CI: 1.003-1.012; $P=0.001$ ) after adjusting for diabetes mellitus, age, hypertension, C-reactive protein, and body weight in multivariable logistic regression analysis. Multivariable forward stepwise linear regression analysis also confirmed that the logarithmically transformed FGF-21 level ( $\beta=3.245$, 95\% CI: 1.593-4.987, $P<0.001)$ was an independent predictor of cfPWV values. The area under the receiver operating characteristic (ROC) curve predicting aortic stiffness by the serum FGF-21 level was 0.693 (95\% CI: 0.606-0.771, $P<0.001$ ). Conclusions. Serum FGF-21 level positively correlates with cfPWV and is also an independent predictor of aortic stiffness in maintenance HD patients.

\section{Introduction}

Cardiovascular disease contributes to nearly half of deaths in patients with end-stage renal disease (ESRD) [1]. Patients with ESRD have a more than 10-fold increase in cardiovascular mortality, especially in younger individuals, compared with the general population [2]. Cardiovascular changes include accelerated atherosclerosis and arteriosclerosis with increased arterial stiffness [3]. The measurement of arterial stiffness helps assess cardiovascular risk and the development of therapeutical interventions. The carotidfemoral pulse wave velocity (cfPWV) is the established standard for measuring aortic stiffness, and it predicts cardiovascular and overall mortality in the ESRD population [4].

Fibroblast growth factor 21 (FGF-21) is a predominantly liver-derived hormone involved in regulating glucose and lipid metabolism, acting as a downstream target of peroxisome proliferator-activated receptor (PPAR) $\alpha$ and PPAR $\gamma$. It exerts versatile metabolic actions by binding to the FGF receptor and $\beta$-klotho, an extensively expressed transmembrane protein [5]. FGF-21 modulates gluconeogenesis, ketogenesis, insulin sensitivity, mitochondrial function, thermogenesis, and lipid metabolism [6, 7]. It has favorable metabolic effects in mice $[8,9]$; in humans, however, higher circulating FGF21 concentrations are associated with dyslipidemia and diabetes [10-12]. As a member of the FGF family, FGF-21 levels rise progressively during chronic kidney disease $[11,13]$. Its association with inflammation, arterial stiffness, and increased cardiovascular burden has been reported $[14,15]$, suggesting that FGF-21 has potential as a marker for arterial stiffness in selected populations. Thus, the present study aimed to examine the association of 
FGF-21 and cfPWV in regular hemodialysis (HD) patients.

\section{Materials and Methods}

2.1. Patients. We recruited $130 \mathrm{HD}$ patients from Hualien Tzu Chi Hospital Dialysis Center between March and July 2016. All subjects were over 20 years old and had undergone regular $\mathrm{HD}$ for at least 3 months. High-flux polysulfone disposable artificial kidneys (FX class dialyzer; Fresenius Medical Care, Bad Homburg, Germany) were used in the dialysis unit. Patients with malignancy, stroke, acute infection, amputated limbs, or who were bed-ridden were excluded from the study. Blood pressure was taken before HD sessions. A diagnosis of diabetes mellitus (DM) or hypertension was made if a patient was documented or taking insulin, hypoglycemic, or antihypertensive medications. Medical records were reviewed for the etiology of ESRD, prescription of angiotensin II receptor blockers, calcium channel blockers, $\beta$-blockers, statins, or fibrates. The research was carried out in accordance with the World Medical Association Declaration of Helsinki. The study protocol was approved by the Research Ethics Committee, Hualien Tzu Chi Hospital, Buddhist Tzu Chi Medical Foundation (IRB103-136-B), and informed consent was obtained from each subject.

\subsection{Anthropometric Analysis and Biochemical} Determinations. Height and post-HD body weight were measured, and body mass index (BMI) was calculated $[16,17]$. According to the criteria specified by the Department of Health in Taiwan, overweight was defined as BMI $\geq 24$ and obese as $\mathrm{BMI} \geq 27$ [18]. Fasting blood samples were collected and centrifuged at 3000 RPM for $10 \mathrm{~min}$ before HD. Serum samples were stored at $4^{\circ} \mathrm{C}$ and analyzed within $1 \mathrm{~h}$ of collection. Serum levels of blood urea nitrogen, creatinine, total cholesterol, triglyceride, glucose, total calcium, phosphorus, and C-reactive protein (CRP) were measured by standard laboratory methods (Siemens Advia 1800, Siemens Healthcare GmbH, Henkestr, Germany) [16, 17]. The fractional clearance index for urea $(\mathrm{Kt} / \mathrm{V})$ was calculated from a formal, single-compartment dialysis urea kinetic model. Serum FGF-21 (Phoenix Pharmaceuticals, Inc., Burlingame, CA, USA) concentrations were measured with commercially available enzyme immunoassay kits [19]. Serum intact parathyroid hormone (iPTH) levels were measured by the enzyme-linked immunosorbent assay (Abcam, Cambridge, MA, USA) [16, 17].

\subsection{Carotid-Femoral Pulse Wave Velocity Measurements.} cfPWV values were obtained by transcutaneous recording of the pressure pulse waveform in the target artery using applanation tonometry (SphygmoCor system, AtCor Medical, NSW, Australia). These measurements were performed with the patient in the supine position after a 10-min rest in a quiet, temperature-controlled room with simultaneous electrocardiographic (ECG) recordings. Pulse wave recordings were obtained consecutively at two superficial artery sites (carotid-femoral segment). Each set of pulse waves was processed with the Integral software (SphygmoCor system, AtCor Medical, NSW, Australia). ECG data were used to calculate the mean time difference between successive R-waves and pulse waves for each beat over an average of 10 consecutive cardiac cycles. The carotid-femoral distance was determined by subtracting the distance from the carotid location to the suprasternal notch, from the distance between the suprasternal notch and the femoral site. The cfPWV was calculated by dividing the distance by the mean time difference between the two recorded points. Quality indices were set to ensure data uniformity. Increased central arterial stiffness was defined as a cfPWV value $>10 \mathrm{~m} / \mathrm{s}$, according to the 2018 European Society of Cardiology guidelines, as a conservative cutoff for an increased cardiovascular risk [20].

2.4. Statistical Analysis. All statistical analyses were done with SPSS (version 19.0; SPSS Inc., Chicago, IL, USA). The Kolmogorov-Smirnov test was performed to determine the normal distribution. Normally distributed data are expressed as the mean \pm standard deviation, and differences between groups were evaluated by the independent samples $t$-test. Non-normally-distributed data are expressed as medians and interquartile ranges. The differences between groups were evaluated by Mann-Whitney $U$ test. Categorical data were tested by the chi-square test and are expressed as frequencies (\%). Skewed distributions were transformed using base-10 logarithms (log) for analysis. The correlation between clinical variables and cfPWV values was evaluated using simple linear regression analysis, and variables that were significantly correlated with cfPWV values were tested for independence using a multivariable forward stepwise regression analysis. Variables significantly associated with aortic stiffness were tested for independence by multivariate logistic regression analysis. A receiver operating characteristic (ROC) curve was used to calculate the area under the curve (AUC) to identify the FGF-21 level that predicts aortic stiffness in HD patients. $P<0.05$ was considered statistically significant.

\section{Results}

3.1. Baseline Characteristics. The demographic and biochemical characteristics of the participants are summarized in Table 1. A total of 130 subjects, including 76 patients with a normal cfPWV (53.9\% females, mean age $=61.8 \pm 13.7$ years) and 54 subjects with an increased cfPWV $(42.6 \%$ females, mean age $=66.7 \pm 12.0$ years), were included. The etiologies of ESRD were diabetic nephropathy in 49 patients (37.7\%), glomerulonephritis in 54 patients (41.5\%), hypertensive nephropathy in 6 patients $(4.6 \%)$, and other causes in 21 patients $(16.2 \%)$. There were no differences in gender, $\mathrm{HD}$ duration, BMI in the obese or overweight range, history of smoking, Kt/V, diastolic blood pressure, and the use of antihypertensive or antihyperlipidemic therapy between the two groups. The blood urea nitrogen, creatinine, total cholesterol, triglycerides, fasting glucose, total calcium, phosphorus, and iPTH levels were similar between the two 
TABLE 1: Clinical characteristics of the study population.

\begin{tabular}{|c|c|c|c|c|}
\hline Parameters & Overall $(n=130)$ & $\mathrm{cfPWV} \leq 10 \mathrm{~m} / \mathrm{s}(n=76)$ & $\mathrm{cfPWV}>10 \mathrm{~m} / \mathrm{s}(n=54)$ & $P$ value \\
\hline \multicolumn{5}{|l|}{ Clinical characteristics } \\
\hline Age (years) & $63.8 \pm 13.2$ & $61.8 \pm 13.7$ & $66.7 \pm 12.0$ & $0.036^{*}$ \\
\hline Hemodialysis duration (months) & $59.6(23.9-130.7)$ & $80.0(22.2-144.8)$ & $56.5(26.7-92.9)$ & 0.232 \\
\hline Female, $n(\%)$ & $64(49.2)$ & $41(53.9)$ & $23(42.6)$ & 0.202 \\
\hline Smoking, $n(\%)$ & $18(13.8)$ & $8(10.5)$ & $10(18.5)$ & 0.303 \\
\hline Body weight $(\mathrm{kg})$ & $63.2 \pm 14.6$ & $60.8 \pm 14.1$ & $66.5 \pm 14.7$ & $0.027^{*}$ \\
\hline Body mass index $\left(\mathrm{kg} / \mathrm{m}^{2}\right)$ & $24.7 \pm 4.8$ & $24.0 \pm 4.7$ & $25.7 \pm 4.9$ & $0.048^{*}$ \\
\hline Overweight or obese & $67(51.5)$ & $37(48.7)$ & $30(55.6)$ & 0.480 \\
\hline $\mathrm{Kt} / \mathrm{V}$ (gotch) & $1.3 \pm 0.2$ & $1.4 \pm 0.2$ & $1.3 \pm 0.2$ & 0.200 \\
\hline \multicolumn{5}{|l|}{ Hemodynamic parameters } \\
\hline Carotid-femoral PWV (m/s) & $10.1 \pm 3.3$ & $7.8 \pm 1.2$ & $13.2 \pm 2.6$ & $<0.001^{*}$ \\
\hline Systolic blood pressure (mmHg) & $142.0 \pm 25.9$ & $138.2 \pm 26.7$ & $147.4 \pm 23.8$ & $0.044^{*}$ \\
\hline Diastolic blood pressure $(\mathrm{mmHg})$ & $77.0 \pm 15.7$ & $77.5 \pm 15.6$ & $76.2 \pm 16.0$ & 0.658 \\
\hline Mean arterial pressure $(\mathrm{mmHg})$ & $98.6 \pm 17.8$ & $97.7 \pm 18.2$ & $100.0 \pm 17.2$ & 0.478 \\
\hline Pulse pressure $(\mathrm{mmHg})$ & $65.0 \pm 18.0$ & $60.7 \pm 17.4$ & $71.2 \pm 17.0$ & $0.001^{*}$ \\
\hline \multicolumn{5}{|l|}{ Laboratory parameters } \\
\hline Total cholesterol (mg/dL) & $146.8 \pm 34.6$ & $148.3 \pm 39.0$ & $144.5 \pm 27.6$ & 0.535 \\
\hline Triglyceride $(\mathrm{mg} / \mathrm{dL})$ & $114.0(84.0-187.0)$ & $108.5(83.3-186.5)$ & $122.0(87.0-187.5)$ & 0.406 \\
\hline Fasting glucose $(\mathrm{mg} / \mathrm{dL})$ & $130.5(109.8-167.3)$ & $128.0(106.3-151.0)$ & $133.5(110.8-182.0)$ & 0.135 \\
\hline Blood urea nitrogen $(\mathrm{mg} / \mathrm{dL})$ & $60.83 \pm 15.00$ & $61.72 \pm 14.48$ & $59.57 \pm 15.76$ & 0.423 \\
\hline Creatinine $(\mathrm{mg} / \mathrm{dL})$ & $9.31 \pm 2.07$ & $9.42 \pm 2.00$ & $9.14 \pm 2.18$ & 0.456 \\
\hline Total calcium (mg/dL) & $9.0 \pm 0.8$ & $8.9 \pm 0.7$ & $9.2 \pm 0.8$ & 0.067 \\
\hline Phosphorus (mg/dL) & $4.8 \pm 1.3$ & $4.7 \pm 1.3$ & $4.8 \pm 1.3$ & 0.708 \\
\hline iPTH $(\mathrm{pg} / \mathrm{mL})$ & $204.1(69.4-462.7)$ & $256.45(106.4-475.2)$ & $150.7(51.2-462.7)$ & 0.163 \\
\hline C-reactive protein $(\mathrm{mg} / \mathrm{dL})$ & $0.26(0.08-0.66)$ & $0.23(0.06-0.46)$ & $0.38(0.12-0.95)$ & $0.040^{*}$ \\
\hline FGF-21 (pg/mL) & $1150.5(728.7-1863.1)$ & $887.5(572.3-1579.8)$ & $1433.1(889.3-2317.7)$ & $<0.001^{*}$ \\
\hline \multicolumn{5}{|l|}{ Underlying disease } \\
\hline Diabetes mellitus, $n(\%)$ & $49(37.7)$ & $18(23.7)$ & $31(57.4)$ & $<0.001^{*}$ \\
\hline Hypertension, $n(\%)$ & $62(47.7)$ & $30(39.5)$ & $32(59.3)$ & $0.026^{*}$ \\
\hline \multicolumn{5}{|l|}{ Etiology of ESRD } \\
\hline Diabetic nephropathy, $n(\%)$ & $49(37.7)$ & $18(23.7)$ & $31(57.4)$ & $<0.001^{*}$ \\
\hline Hypertensive nephropathy, $n(\%)$ & $6(4.6)$ & $4(5.3)$ & $2(3.7)$ & 1.000 \\
\hline Glomerulonephritis, $n(\%)$ & $54(41.5)$ & $38(50.0)$ & $16(29.6)$ & $0.030^{*}$ \\
\hline Other, $n(\%)$ & $21(16.2)$ & $16(21.1)$ & $5(9.3)$ & 0.092 \\
\hline \multicolumn{5}{|l|}{ Medication } \\
\hline ACE-inhibitors/ARBs, $n(\%)$ & $38(29.2)$ & $19(25.0)$ & $19(35.2)$ & 0.208 \\
\hline$\beta$-blocker, $n(\%)$ & $36(27.7)$ & $19(25.0)$ & $17(31.5)$ & 0.416 \\
\hline Calcium channel blocker, $n(\%)$ & $50(38.5)$ & $30(39.5)$ & $20(37.0)$ & 0.778 \\
\hline Statin, $n(\%)$ & $20(15.4)$ & $9(11.8)$ & $11(20.4)$ & 0.184 \\
\hline Fibrate, $n(\%)$ & $15(11.5)$ & $8(10.5)$ & $7(13.0)$ & 0.668 \\
\hline
\end{tabular}

Values for continuous variables are shown as mean \pm standard deviation after analysis by student's $t$-test; variables not normally distributed are shown as median and interquartile range after analysis by Mann-Whitney $U$ test; values are presented as number (\%)after analysis by the chi-square test. Kt/V, fractional clearance index for urea; cfPWV, carotid-femoral pulse wave velocity; iPTH, intact parathyroid hormone; FGF-21, fibroblast growth factor 21; ESRD, end-stage renal disease; ACE, angiotensin-converting enzyme; ARB, angiotensin receptor blockers. ${ }^{*} P<0.05$ was considered statistically significant.

groups. Compared with subjects who had a normal cfPWV, subjects with an increased cfPWV were significantly older $(P=0.036)$, had a higher body weight $(P=0.027)$, BMI $(P=0.048)$, systolic blood pressure $(P=0.044)$, pulse pressure $(P=0.001)$, CRP $(P=0.040)$, FGF-21 $(P<0.001)$ (Figure 1$)$, and a higher rate of DM $(P<0.001)$ and hypertension $(P=0.026)$. Subjects with underlying diabetic nephropathy were more likely to have increased PWV $(P<0.001)$, while subjects with underlying glomerulonephritis were more likely to have normal PWV $(P=0.030)$
3.2. Serum FGF-21 Was Independently Associated with an Increased cfPWV $(>10 \mathrm{~m} / \mathrm{s})$. To determine the independent predictors for an increased cfPWV, we performed multivariable logistic regression analysis to determine the associations of FGF-21 and aortic stiffness (Table 2). FGF-21 was independently associated with an increased cfPWV (per $10 \mathrm{pg} / \mathrm{mL}$, odds ratio (OR): $1.008 ; 95 \%$ confidence interval (CI): 1.003-1.012; $P=0.001$ ) after adjusting for other factors associated with increased cfPWV identified from Table 1 (DM, hypertension, age, CRP, and body weight). DM was also a predictor of increased cfPWV (OR: 4.269; 95\% CI: 


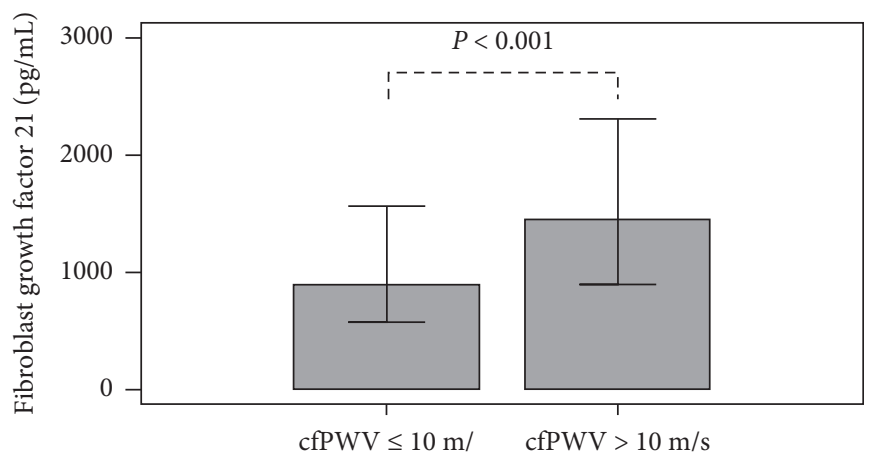

FIgURE 1: Comparison of fibroblast growth factor 21 levels between 130 hemodialysis patients with or without aortic stiffness.

TABLE 2: Multivariable logistic regression analysis of the factors correlated to aortic stiffness.

\begin{tabular}{lccc}
\hline Variables & Odds ratio & 95\% confidence interval & $P$ value \\
\hline FGF-21, per $10 \mathrm{pg} / \mathrm{mL}$ & 1.008 & $1.003-1.012$ & $0.001^{*}$ \\
Diabetes mellitus, present & 4.269 & $1.768-10.309$ & $0.001^{*}$ \\
Age, per 10 years & 1.412 & $0.986-2.024$ & 0.060 \\
Hypertension, present & 3.105 & $1.279-7.540$ & 0.012 \\
C-reactive protein, $1 \mathrm{mg} / \mathrm{dL}$ & 2.103 & $0.994-4.446$ & 0.052 \\
Body weight, $1 \mathrm{~kg}$ & 1.027 & $0.995-1.061$ & 0.093 \\
\hline
\end{tabular}

Analysis of data was performed using the multivariate logistic regression analysis (adopted factors: diabetes mellitus, hypertension, age, body weight, Creactive protein, and FGF-21). FGF-21, fibroblast growth factor $21 .{ }^{*} P<0.05$ was considered statistically significant.

1.768-10.309; $P=0.001)$. ROC analysis was performed to identify the cutoff values of FGF-21 for aortic stiffness, and the AUC was $0.693(P<0.001)$ (Figure 2), and at an FGF-21 cutoff level of $1063.5 \mathrm{pg} / \mathrm{mL}$, the sensitivity was $70 \%$ and the specificity was $40 \%$.

3.3. Correlations between cfPWV Levels and Clinical Variables. We analyzed the correlations between the cfPWV values and clinical parameters (Table 3 ). The cfPWV value was positively correlated with age $(r=0.194, P=0.027)$, systolic blood pressure $(r=0.186, P=0.034)$, pulse pressure $(r=0.272, \quad P=0.002)$, logarithmically transformed CRP (log-CRP, $r=0.242, \quad P=0.006), \quad \log -\mathrm{FGF}-21 \quad(r=0.335$, $P<0.001)$, and the presence of DM $(r=0.378, P<0.001)$. Furthermore, the stepwise multivariable regression analysis showed the log-FGF-21 level to be an independent predictor for $\operatorname{cfPWV}(\beta=3.245$; 95\% CI: 1.593-4.987, $P<0.001$, Table 3$)$, among others including DM status $(\beta=1.974 ; 95 \% \mathrm{CI}$ : $0.918-3.031, P=0.001)$ and log-CRP levels $(\beta=0.978 ; 95 \%$ CI: $0.030-1.927, P=0.037)$. All other tested variables were not significant and were excluded.

\section{Discussion}

The most important finding of this study was the association of FGF-21 levels and cfPWV values, shown for the first time in regular $\mathrm{HD}$ patients.

Patients suffering from ESRD have increased arterial stiffness and cardiovascular mortality [21-23], resulting from traditional and nontraditional uremia-related cardiovascular risk factors. cfPWV has been reported as a strong independent predictor of overall and cardiovascular mortality in a hemodialysis population [4]. In general, arteries

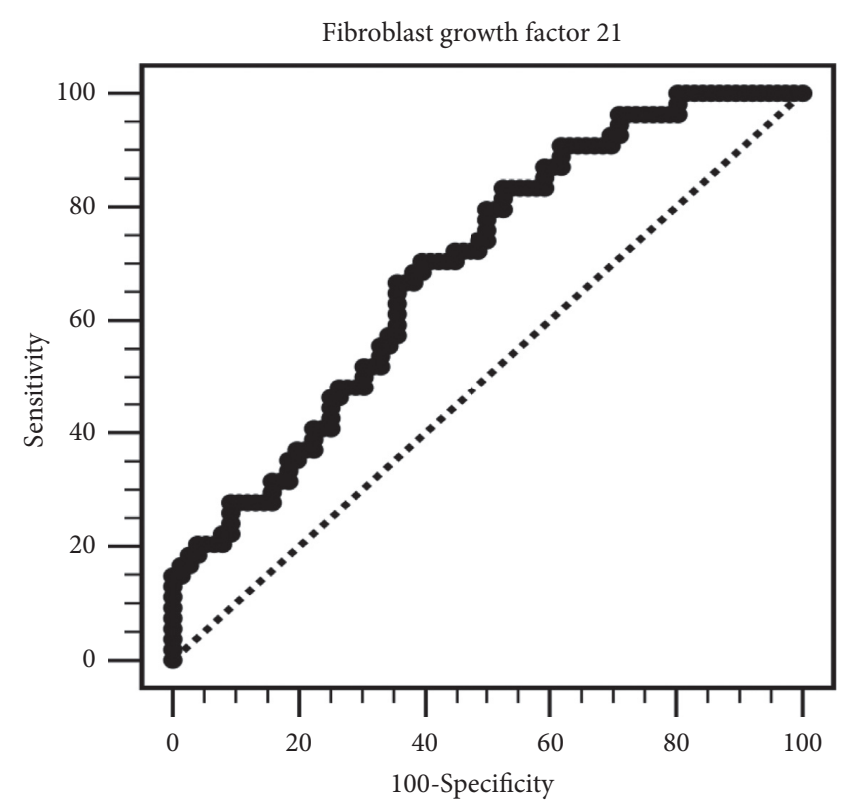

Figure 2: The area under the receiver operating characteristic curve indicates the predictive power of fibroblast growth factor 21 for aortic stiffness.

become stiffer with age and may develop atherosclerosis. The pathogenesis involves structural changes in the medial layer of the elastic arteries, such as the aorta and other major arteries. There is endothelial and smooth muscle dysfunction caused by inflammation and increased oxidative stress [21]. The most prevalent vascular findings are luminal narrowing, wall thickening, and reduced elasticity, that is, arteriosclerosis [24]. 
TABLE 3: Correlations between central pulse wave velocity and clinical variables and multivariable stepwise linear regression analysis of cfPWV.

\begin{tabular}{|c|c|c|c|c|c|}
\hline \multirow{3}{*}{ Variables } & \multicolumn{5}{|c|}{ Carotid-femoral pulse wave velocity $(\mathrm{m} / \mathrm{s})$} \\
\hline & \multicolumn{2}{|c|}{ Simple linear regression } & \multicolumn{3}{|c|}{ Multivariable linear regression } \\
\hline & $r$ & $P$ value & $\beta$ & 95\% confidence interval & $P$ value \\
\hline Female & -0.113 & 0.200 & - & - & - \\
\hline Diabetes mellitus & 0.378 & $<0.001^{*}$ & 1.974 & $0.918-3.031$ & $0.001^{*}$ \\
\hline Hypertension & 0.128 & 0.145 & - & - & - \\
\hline Age (years) & 0.194 & $0.027^{*}$ & - & - & - \\
\hline Log-HD duration (months) & -0.060 & 0.494 & - & - & - \\
\hline Body weight $(\mathrm{kg})$ & 0.162 & 0.066 & - & - & - \\
\hline Body mass index $\left(\mathrm{kg} / \mathrm{m}^{2}\right)$ & 0.133 & 0.131 & - & - & - \\
\hline Systolic blood pressure (mmHg) & 0.186 & $0.034^{*}$ & - & - & - \\
\hline Diastolic blood pressure (mmHg) & -0.004 & 0.966 & - & - & - \\
\hline Mean arterial pressure $(\mathrm{mmHg})$ & 0.088 & 0.320 & & - & - \\
\hline Pulse pressure $(\mathrm{mmHg})$ & 0.272 & $0.002^{*}$ & & - & - \\
\hline Total cholesterol (mg/dL) & -0.039 & 0.663 & - & - & - \\
\hline Log-triglyceride $(\mathrm{mg} / \mathrm{dL})$ & 0.087 & 0.328 & - & - & - \\
\hline Log-fasting plasma glucose $(\mathrm{mg} / \mathrm{dL})$ & 0.110 & 0.213 & - & - & - \\
\hline Total calcium $(\mathrm{mg} / \mathrm{dL})$ & 0.116 & 0.189 & - & - & - \\
\hline Phosphorus (mg/dL) & 0.056 & 0.529 & - & - & - \\
\hline Log-iPTH $(\mathrm{pg} / \mathrm{mL})$ & -0.153 & 0.082 & - & - & - \\
\hline $\log -\mathrm{CRP}(\mathrm{mg} / \mathrm{dL})$ & 0.242 & $0.006^{*}$ & 0.978 & $0.030-1.927$ & $0.037^{*}$ \\
\hline Log-FGF-21 (pg/mL) & 0.335 & $<0.001^{*}$ & 3.245 & $1.593-4.987$ & $<0.001^{*}$ \\
\hline $\mathrm{Kt} / \mathrm{V}$ (gotch) & -0.070 & 0.435 & - & - & - \\
\hline
\end{tabular}

The data of HD duration, triglyceride, fasting plasma glucose, iPTH, CRP, and FGF-21 levels showed skewed distribution and therefore were log-transformed before analysis. Analysis of data was performed using the simple linear regression analysis or multivariate stepwise linear regression analysis (adopted factors: diabetes mellitus, age, systolic blood pressure, pulse pressure, log-CRP, and log-FGF-21). HD, hemodialysis; iPTH, intact parathyroid hormone; CRP, Creactive protein; FGF-21, fibroblast growth factor $21 ; \mathrm{Kt} / \mathrm{V}$, fractional clearance index for urea; $\beta$, unstandardized regression coefficient. ${ }^{*} P<0.05$ was considered statistically significant.

Factors related to diseases such as DM, hypertension, and chronic kidney disease and lifestyle habits such as smoking contribute to arterial stiffness beyond normal aging [21]. Advanced chronic kidney disease is associated with worse arterial stiffness than early disease [25]. Accelerated progression of arterial stiffness has been noted in the dialysis population; uremic factors, in addition to traditional risk factors, have been identified as independent determinants of this progression [26]. In line with the abovementioned reports, this study showed that aortic stiffness was correlated with DM, increased systolic blood pressure, pulse pressure, $\mathrm{CRP}$, and age in regular HD patients.

Blood pressure is one of the most significant contributing factors to cfPWV [27]. In this study, cfPWV is correlated with systolic blood pressure and pulse pressure but not diastolic blood pressure or mean arterial pressure, showing that pulsatile blood pressure had more impact on cfPWV than the steady component. Similarly, a previous invasive study showed that pulse pressure had the best correlation with cfPWV among a group of normotensive and hypertensive subjects [28]. This increased pulsatility transmits to low resistance vascular beds, causing increased vascular mechanical strain and subsequent organ damage [29]. In our study, the association between cfPWV and age, systolic blood pressure, and pulse pressure disappeared after multivariate linear regression analysis, suggesting that DM, inflammation, and FGF-21 are more important determinants of arterial stiffness in the ESRD population with accelerated and deranged vascular aging.
Studies have shown that exogenous FGF-21 analog administration can significantly improve cardiometabolic profiles in obese or overweight diabetic patients [30-32]. This protective effect on the cardiovascular system is consistent with the beneficial effects of FGF-21 observed in animal studies. Nevertheless, the elevation of serum FGF-21 is associated with several vascular complications, including increased intima-media thickness, atherosclerotic plaque formation, and arterial stiffness [10, 15, 33, 34]. Whether the elevation of FGF-21 is beneficial or harmful to the cardiovascular system remains unclear. This paradoxical elevation could be a compensatory response to the underlying cardiovascular stress or FGF-21 resistance caused by the downregulation of target organ receptors [32, 35, 36]. Therefore, FGF-21 might serve as a potential marker for cardiovascular stress.

Studies showed that FGF-21 exerts protective effects against the development of atherosclerosis through the modulation of interactions between the adipose tissue, liver, and blood vessels [37], reducing vascular inflammation and oxidative stress [38]. In apolipoprotein E-/- mice, FGF-21 deficiency results in accelerated atherosclerosis and premature death, along with hypoadiponectinemia and hypercholesterolemia. Exogenous treatment with recombinant mouse FGF-21 induces adipocyte secretion of adiponectin, significantly reducing neointima formation, the proliferation of smooth muscle cells, and macrophage inflammation [37]. Hypercholesterolemia was also diminished via downregulation of the transcription factor sterol regulatory 
element-binding protein-2 in hepatocytes [37]. FGF-21 inhibits NLRP3 inflammasome-mediated vascular endothelial cells pyroptosis, possibly by improving mitochondrial function, reducing ROS production, and endoplasmic reticulum stress in the endothelial cells $[39,40]$. An in vitro study revealed that FGF-21 protects macrophages against ox-LDL-induced foam cell formation and apoptosis by suppressing the CHOP expression [41]. Overall, FGF-21 exerts beneficial vascular effects, possibly by mitigating vascular inflammation and dyslipidemia, independent of its antiobese and antidiabetic activity. Hence, elevated serum FGF-21 associated with atherosclerosis likely reflects this adaptive mechanism $[37,38]$. In patients with ESRD, serum FGF-21 levels may be up to 20 times normal levels [11]. FGF21 is not dialyzable [42], suggesting that renal factors influence its serum level in ESRD patients. Higher levels of FGF-21 were associated with increased arterial stiffness as measured by flow-mediated dilatation in a study of patients undergoing continuous ambulatory peritoneal dialysis [14]. Moreover, higher FGF-21 levels were associated with a higher all-cause mortality rate but not cardiovascular events, in a Japanese study of HD patients [43].

Our study results should be interpreted considering several limitations. First, we cannot assume any causal associations due to the cross-sectional study design. Second, the sample size was small, and larger longitudinal studies will be needed for confirmation. Third, we only investigated the relationship between cfPWV and FGF-21. Other factors of note, such as intima-media thickness or left ventricular mass index, may provide additional invaluable information. Fourth, we did not measure residual renal function, which may influence the development of atherosclerosis. Fifth, a dietary survey may be needed since FGF-21 is maximally elevated under low protein and high carbohydrate intakes [44]. Sixth, there was no healthy control group for comparison.

\section{Conclusion}

Higher serum FGF-21 levels were associated with higher cfPWV values in a group of regular HD patients. This study suggests that serum FGF-21 levels may be a predictive marker of aortic stiffness in HD patients. Defining this new parameter might help stratify the cardiovascular disease risk and offer potential therapeutic strategies for atherosclerosis. Future studies such as interventional trials are warranted to determine the clinical significance of FGF-21.

\section{Data Availability}

The data used to support the findings of this study are available from the corresponding author on reasonable request.

\section{Disclosure}

The funding source had no role in the conception and conduct of the study; collection, management, analysis, and interpretation of the data; and preparation of the manuscript.

\section{Conflicts of Interest}

The authors declare that they have no conflicts of interest.

\section{Authors' Contributions}

LT Chiu and CD Hung contributed equally to this study. YL Lin and BG Hsu conceived and designed the experiments. LT Chiu, CD Hung, and L Lin performed the experiments. LT Chiu, YL Lin, and BG Hsu analyzed the data. LT Chiu, CD Hung, YL Lin, and BG Hsu wrote the manuscript. All the authors reviewed and approved the final version of this manuscript.

\section{Acknowledgments}

This work was supported by grants from Tzu Chi Hospital (TCRD107-63 and TCMF-CP 110-02) in Taiwan. The authors would like to thank Enago (https://www.enago.tw) for the English language review.

\section{References}

[1] D. J. de Jager, D. C. Grootendorst, K. J. Jager et al., "Cardiovascular and noncardiovascular mortality among patients starting dialysis," JAMA, vol. 302, no. 16, pp. 1782-1789, 2009.

[2] R. Foley, P. Parfrey, and M. Sarnak, "Clinical epidemiology of cardiovascular disease in chronic renal disease," American Journal of Kidney Diseases, vol. 32, no. 5, pp. S112-S119, 1998.

[3] M. J. Sarnak and R. N. Foley, "Cardiovascular mortality in the general population versus dialysis: a glass half full or empty?" American Journal of Kidney Diseases, vol. 58, no. 1, pp. 4-6, 2011.

[4] J. Blacher, A. P. Guerin, B. Pannier, S. J. Marchais, M. E. Safar, and G. M. London, "Impact of aortic stiffness on survival in end-stage renal disease," Circulation, vol. 99, no. 18, pp. 2434-2439, 1999.

[5] A. C. Adams, C. C. Cheng, T. Coskun, and A. Kharitonenkov, "FGF21 requires betaklotho to act in vivo," PLoS One, vol. 7, no. 11, Article ID e49977, 2012.

[6] D. Cuevas-Ramos, R. Mehta, and C. A. Aguilar-Salinas, "Fibroblast growth factor 21 and browning of white adipose tissue," Frontiers in Physiology, vol. 10, Article ID 37, 2019.

[7] M. D. L. Chau, J. Gao, Q. Yang, Z. Wu, and J. Gromada, "Fibroblast growth factor 21 regulates energy metabolism by activating the AMPK-SIRT1-PGC- $1 \alpha$ pathway," Proceedings of the National Academy of Sciences, vol. 107, no. 28, pp. 12553-12558, 2010.

[8] M. K. Badman, P. Pissios, A. R. Kennedy, G. Koukos, J. S. Flier, and E. Maratos-Flier, "Hepatic fibroblast growth factor 21 is regulated by $\operatorname{PPAR} \alpha$ and is a key mediator of hepatic lipid metabolism in ketotic states," Cell Metabolism, vol. 5, no. 6, pp. 426-437, 2007.

[9] A. Kharitonenkov, T. L. Shiyanova, A. Koester et al., "FGF-21 as a novel metabolic regulator," Journal of Clinical Investigation, vol. 115, no. 6, pp. 1627-1635, 2005.

[10] H. Staiger, M. Keuper, L. Berti, M. Hrabě de Angelis, and H.-U. Häring, "Fibroblast growth factor 21-metabolic role in mice and men," Endocrine Reviews, vol. 38, no. 5, pp. 468-488, 2017.

[11] P. G. D. A. Suassuna, R. B. de Paula, H. Sanders-Pinheiro, O. W. Moe, and M.-C. Hu, "Fibroblast growth factor 21 in 
chronic kidney disease," Journal of Nephrology, vol. 32, no. 3, pp. 365-377, 2019.

[12] A. O. Chavez, M. Molina-Carrion, M. A. Abdul-Ghani, F. Folli, R. A. DeFronzo, and D. Tripathy, "Circulating fibroblast growth factor-21 is elevated in impaired glucose tolerance and type 2 diabetes and correlates with muscle and hepatic insulin resistance," Diabetes Care, vol. 32, no. 8, pp. 1542-1546, 2009.

[13] C. Crasto, R. D. Semba, K. Sun, and L. Ferrucci, "Serum fibroblast growth factor 21 is associated with renal function and chronic kidney disease in community-dwelling adults," Journal of the American Geriatrics Society, vol. 60, no. 4, pp. 792-793, 2012.

[14] S. M. Ulu, S. Yuksel, A. Altuntaş et al., “Associations between serum hepcidin level, FGF-21 level and oxidative stress with arterial stiffness in CAPD patients," International Urology and Nephrology, vol. 46, no. 12, pp. 2409-2414, 2014.

[15] S. Yafei, F. Elsewy, E. Youssef, M. Ayman, and M. El-Shafei, "Fibroblast growth factor 21 association with subclinical atherosclerosis and arterial stiffness in type 2 diabetes," $D i$ abetes \& Metabolic Syndrome: Clinical Research Reviews, vol. 13, no. 1, pp. 882-888, 2019.

[16] Y. H. Lai, C. H. Wang, C. H. Kuo, U.-L. Lin, J.-P. Tsai, and B.-G. Hsu, "Serum p-cresyl sulfate level is positively associated with central arterial stiffness in patients on hemodialysis," Toxins, vol. 12, no. 1, Article ID 10, 2019.

[17] J. S. Hou, C. H. Wang, Y. H. Lai et al., "Serum malondialdehyde-modified low-density lipoprotein level is a risk factor for aortic stiffness in maintenance hemodialysis patients," Nutrients, vol. 12, no. 7, Article ID 2160, 2020.

[18] N.-F. Chu, "Prevalence of obesity in taiwan," Obesity Reviews, vol. 6, no. 4, pp. 271-274, 2005.

[19] Y. T. Wu, B. G. Hsu, C. H. Wang, Y.-L. Lin, Y.-H. Lai, and C.-H. Kuo, "Lower serum fibroblast growth factor 21 levels are associated with normal lumbar spine bone mineral density in hemodialysis patients," International Journal of Environmental Research and Public Health, vol. 17, no. 6, Article ID 1938, 2020.

[20] B. Williams, G. Mancia, W. Spiering et al., "ESC/ESH Guidelines for the management of arterial hypertension," European Heart Journal, vol. 39, no. 33, pp. 3021-3104, 2018.

[21] L. Zanoli, P. Lentini, M. Briet et al., "Arterial stiffness in the heart disease of CKD," Journal of the American Society of Nephrology, vol. 30, no. 6, pp. 918-928, 2019.

[22] E. Cabrera-Fischer, Y. Zocalo, S. Wray, and D. Bia, "Arterial Stiffness in haemodialyzed patients: findings and controversies," Current Hypertension Reviews, vol. 14, no. 2, pp. 100-106, 2018.

[23] J. Blacher, K. Demuth, A. P. Guerin, M. E. Safar, N. Moatti, and G. M. London, "Influence of biochemical alterations on arterial stiffness in patients with end-stage renal disease," Arteriosclerosis, Thrombosis, and Vascular Biology, vol. 18, no. 4, pp. 535-541, 1998.

[24] M. Kanbay, B. Afsar, P. Gusbeth-Tatomir, and A. Covic, "Arterial stiffness in dialysis patients: where are we now?" International Urology and Nephrology, vol. 42, no. 3, pp. 741-752, 2010.

[25] M.-C. Wang, W.-C. Tsai, J.-Y. Chen, and J.-J. Huang, "Stepwise increase in arterial stiffness corresponding with the stages of chronic kidney disease," American Journal of Kidney Diseases, vol. 45, no. 3, pp. 494-501, 2005.

[26] P. Avramovski, P. Janakievska, K. Sotiroski, and A. Sikole, "Accelerated progression of arterial stiffness in dialysis patients compared with the general population," The Korean Journal of Internal Medicine, vol. 28, no. 4, pp. 464-474, 2013.

[27] R. Angoff, R. C. Mosarla, and C. W. Tsao, "Aortic stiffness: epidemiology, risk factors, and relevant biomarkers," Front Cardiovascular Medicine, vol. 8, Article ID 709396, 2021.

[28] E. J. Kim, C. G. Park, J. S. Park et al., "Relationship between blood pressure parameters and pulse wave velocity in normotensive and hypertensive subjects: invasive study," Journal of Human Hypertension, vol. 21, no. 2, pp. 141-148, 2007.

[29] M. E. Safar and P. Lacolley, "Disturbance of macro- and microcirculation: relations with pulse pressure and cardiac organ damage," American Journal of Physiology-Heart and Circulatory Physiology, vol. 293, no. 1, pp. H1-H7, 2007.

[30] G. Gaich, J. Y. Chien, H. Fu et al., "The effects of LY2405319, an FGF21 analog, in obese human subjects with type 2 diabetes," Cell Metabolism, vol. 18, no. 3, pp. 333-340, 2013.

[31] S. Talukdar, Y. Zhou, D. Li et al., "A long-acting FGF21 molecule, PF-05231023, decreases body weight and improves lipid profile in non-human primates and type 2 diabetic subjects," Cell Metabolism, vol. 23, no. 3, pp. 427-440, 2016.

[32] J. Kokkinos, S. Tang, K.-A. Rye, and K. L. Ong, "The role of fibroblast growth factor 21 in atherosclerosis," Atherosclerosis, vol. 257, pp. 259-265, 2017.

[33] S.-Y. An, M. S. Lee, S.-A. Yi et al., "Serum fibroblast growth factor 21 was elevated in subjects with type 2 diabetes mellitus and was associated with the presence of carotid artery plaques," Diabetes Research and Clinical Practice, vol. 96, no. 2, pp. 196-203, 2012.

[34] W. S. Chow, A. Xu, Y. C. Woo et al., "Serum fibroblast growth factor-21 levels are associated with carotid atherosclerosis independent of established cardiovascular risk factors," Arteriosclerosis, Thrombosis, and Vascular Biology, vol. 33, no. 10, pp. 2454-2459, 2013.

[35] F. M. Fisher, P. C. Chui, P. J. Antonellis et al., "Obesity is a fibroblast growth factor 21 (FGF21)-resistant state," Diabetes, vol. 59, no. 11, pp. 2781-2789, 2010.

[36] A. Salminen, K. Kaarniranta, and A. Kauppinen, "Integrated stress response stimulates FGF21 expression: systemic enhancer of longevity," Cellular Signalling, vol. 40, pp. 10-21, 2017.

[37] Z. Lin, X. Pan, F. Wu et al., "Fibroblast growth factor 21 prevents atherosclerosis by suppression of hepatic sterol regulatory element-binding protein- 2 and induction of adiponectin in mice," Circulation, vol. 131, no. 21, pp. 1861-1871, 2015.

[38] F. S. Tabari, A. Karimian, H. Parsian et al., "The roles of FGF21 in atherosclerosis pathogenesis," Reviews in Endocrine \& Metabolic Disorders, vol. 20, no. 1, pp. 103-114, 2019.

[39] Z. Zeng, Q. Zheng, J. Chen et al., "FGF21 mitigates atherosclerosis via inhibition of NLRP3 inflammasome-mediated vascular endothelial cells pyroptosis," Experimental Cell Research, vol. 393, no. 2, Article ID 112108, 2020.

[40] W. Wei, X.-X. Li, and M. Xu, "Inhibition of vascular neointima hyperplasia by FGF21 associated with FGFR1/Syk/ NLRP3 inflammasome pathway in diabetic mice," Atherosclerosis, vol. 289, pp. 132-142, 2019.

[41] E. Li, T. Wang, F. Wang et al., "FGF21 protects against oxLDL induced apoptosis through suppressing CHOP expression in THP1 macrophage derived foam cells," BMC Cardiovascular Disorders, vol. 15, Article ID 80, 2015.

[42] J. Hindricks, T. Ebert, A. Bachmann et al., "Serum levels of fibroblast growth factor-21 are increased in chronic and acute renal dysfunction," Clinical Endocrinology, vol. 80, no. 6, pp. 918-924, 2014. 
[43] M. Kohara, T. Masuda, K. Shiizaki et al., "Association between circulating fibroblast growth factor 21 and mortality in endstage renal disease," PLoS One, vol. 12, no. 6, Article ID e0178971, 2017.

[44] S. M. Solon-Biet, V. C. Cogger, T. Pulpitel et al., "Defining the nutritional and metabolic context of FGF21 using the geometric framework," Cell Metabolism, vol. 24, no. 4, pp. $555-565,2016$. 